\title{
Primary peritoneal drainage in neonates with necrotizing enterocolitis associated with congenital heart disease: a single experience in a Brazilian tertiary center
}

\author{
W.C. Canesin ${ }^{1 \oplus}$, F.A.P. Volpe ${ }^{1 \oplus}$, W.A. Gonçalves-Ferri ${ }^{2 \oplus}$, P.H. Manso ${ }^{2 \oplus}$, D.C. Aragon ${ }^{2 \oplus}$, \\ and L. Sbragia ${ }^{10 \times}$ \\ ${ }^{1}$ Laboratório de Cirurgia Experimental Fetal "Michael Harrison”, Divisão de Cirurgia Pediátrica, Departamento de Cirurgia e \\ Anantomia, Faculdade de Medicina de Ribeirão Preto, Universidade de São Paulo, Ribeirão Preto, SP, Brasil \\ ${ }^{2}$ Departamento de Pediatria, Faculdade de Medicina de Ribeirão Preto, Universidade de São Paulo, Ribeirão Preto, SP, Brasil
}

\begin{abstract}
Necrotizing enterocolitis (NEC) is a common condition in preterm infants. The risk factors that contribute to NEC include asphyxia, apnea, hypotension, sepsis, and congenital heart diseases (CHD). The objective of this study was to evaluate the association between the treatment (surgery or drainage) and unfavorable outcomes in neonates with NEC and congenital heart diseases (NEC + CHD). A 19-year retrospective cohort study was conducted (2000-2019). Inclusion criterion was NEC Bell II stage. Exclusion criteria were associated malformation or genetic syndrome and those who did not undergo echocardiography or had a Bell I diagnosis. We included 100 neonates: NEC $(n=52)$ and NEC + CHD $(n=48)$. The groups were subdivided into NEC patients undergoing surgery (NECS, $n=31$ ), NEC patients undergoing peritoneal drainage $(N E C D, n=19), N E C+C H D$ patients undergoing surgery (NECCAS, $n=21$ ), and NEC + CHD patients who were drained (NECCAD, $n=29$ ). Multivariate analysis was performed to estimate the relative risk of death and the length of stay. Covariates were birth weight and gestational age. The group characteristics were similar. The adjusted relative risk of death was higher in the drainage groups [NECD (Adj $\mathrm{RR}=2.70(95 \% \mathrm{Cl}: 1.47 ; 4.97)$ and NECCAD (Adj RR=1.97 $(95 \% \mathrm{Cl}: 1.08 ; 3.61)]$, and they had the shortest time to death: $\mathrm{NECD}=8.72(95 \% \mathrm{Cl}: 3.10 ; 24.54)$ and $\mathrm{NECCAD}=5.32(95 \% \mathrm{Cl}: 1.95 ; 14.44)$. We concluded that performing primary peritoneal drainage in neonates with or without CHD did not improve the number of days of life, did not decrease the risk of death, and was associated with a higher mortality in newborns with NEC and clinical instability.
\end{abstract}

Key words: Necrotizing enterocolitis; Peritoneal drainage; Congenital heart diseases

\section{Introduction}

Necrotizing enterocolitis (NEC) is the most common reason for neonatal abdominal surgery. The overall incidence of NEC is approximately 1 in 1000 live births, but its incidence is clearly inversely related to the birth weight and gestational age, and it affects up to $10 \%$ of infants weighing less than $1500 \mathrm{~g}$ (1). NEC has a multifactorial etiology, and an inadequate inflammatory response to some type of insult (congenital heart disease, infection, formula feeding, or combined factors) may culminate in intestinal necrosis and perforation (2).

Full-term infants who develop NEC usually have other associated factors that predispose them to the disease, such as sepsis, low Apgar scores, prolonged rupture of membranes, exchange transfusions, neural tube defects, and congenital heart disease (CHD) (3). Patients with CHD have a $3.3-11 \%$ risk of developing NEC $(4,5)$, which is much higher relative to other full-term infants. The pathophysiology of NEC in CHD patients remains unknown, but it is theorized that infants with CHD have lower diastolic pressure, which leads to lower bowel perfusion pressures and lower systemic oxygenated blood flow, contributing to an overall state of bowel hypo-perfusion and increased levels of circulating pro-inflammatory cytokines (6).

NEC is initially treated through clinical management, but surgical intervention is required if intestinal perforation or necrosis occurs (7). Mortality by surgical NEC is still approximately $50 \%$, despite all of the scientific improvements in the last few decades (8). However, some authors

Correspondence: L. Sbragia: <sbragia@fmrp.usp.br>

Received August 2, 2020 | Accepted April 26, 2021 
advocate that surgical intervention results in higher survival than medical management in patients with CHD (9). Surgical treatment may be achieved by laparotomy or primary peritoneal drainage (PPD) (10), but which is the best technique is still unclear $(11,12)$. In fact, adequate evidence regarding the best treatment for NEC in babies with hemodynamic instability and patients with patent ductus arteriosus (PDA) or other heart diseases is lacking.

We aimed to evaluate the association between the type of treatment (drainage or surgery) and outcomes in patients with NEC and CHD.

\section{Material and Methods}

This study was approved by the local ethics committee for human research approval number - CEP/Plataforma Brasil CAAE: 16180819.8.0000.5440 (number: 077334/ 2019, Ribeirao Preto Medical School, USP). We retrospectively analyzed the medical charts of newborns hospitalized at a Ribeirão Preto general hospital from 2000 to 2019. The inclusion criterion was a diagnosis of NEC (Bell II or III) (13) made by the neonatology staff. The exclusion criteria were other malformations, a genetic syndrome, those who did not undergo echocardiography, or had a Bell I diagnosis. All of the NEC and NEC + CHD patients were submitted to an echocardiogram performed by the cardiology staff. We considered heart disease to be a PDA $>1 \mathrm{~cm}$ and other congenital heart defects.

The patients were divided into two groups depending on the presence of $\mathrm{CHD}$ (the NEC and $\mathrm{NEC}+\mathrm{CHD}$ groups). The groups were subdivided into NEC undergoing surgery (NECS), NEC undergoing peritoneal drainage (NECD), NEC + CHD undergoing surgery (NECCAS), and $\mathrm{NEC}+\mathrm{CHD}$ undergoing drainage (NECCAD).

In the groups with drainage, the PPD was performed in the lower right quadrant when the babies had pneumoperitoneum and could not be immediately submitted to surgery due to severe thrombocytopenia, hemodynamic instability, bleeding, or shock with a demand for vasoactive drugs. As soon as the baby achieved clinical stability, they would be submitted to laparotomy.

In the groups with surgery, a laparotomy was performed if the baby had pneumoperitoneum or showed no improvement after 15 days of clinical treatment (fasting, abdominal decompression, and broad-spectrum antibiotics).

Chorioamnionitis and maternal hypertension diagnoses were performed by the obstetricians. Small to gestational age was determined by Intergrowth 21 . Gestational age was estimated by the Ballard score for newborns $<37$ weeks and the Capurro score for $\geqslant 37$ weeks.

For the estimation of gross and adjusted relative risks, simple and multiple log-binomial regression models were used. Kaplan-Meier curves were constructed to describe the length of stay. The time until death and the time until discharge from the ICU were evaluated. For the latter, deaths were removed from the database. For the time until death, hazard ratios were estimated by adjusting a Cox proportional hazards model. In this case, weight, gestational age, and heart disease (only for comparisons of 2 groups) were also considered as covariates. For the time until discharge from the ICU, the groups were compared using the Wilcoxon test.

\section{Results}

We included 273 patients with NECII in the period studied, and after applying the exclusion criteria, 173 patients were excluded. We analyzed 100 patients, 48 (48\%) of which had NEC and CHD.

Table 1 shows the characteristics of the groups. We noticed similar characteristics among the groups, although chorioamnionitis was more predominant in the groups with $\mathrm{CHD}$. The length of stay was the same among the groups.

A multivariate analysis was performed to evaluate the association between death and the presence of heart disease and the type of surgical treatment. There was an

Table 1. Characteristics of the studied groups according to the presence of heart disease and type of surgical treatment.

\begin{tabular}{|c|c|c|c|c|c|}
\hline Characteristics & $\begin{array}{l}\text { NECS } \\
N=31\end{array}$ & $\begin{array}{c}\text { NECCAS } \\
\mathrm{N}=19\end{array}$ & $\begin{array}{l}\text { NECD } \\
\mathrm{N}=21\end{array}$ & $\begin{array}{c}\text { NECCAD } \\
\mathrm{N}=29\end{array}$ & $P$ \\
\hline Birth weight in $\mathrm{g}$ (mean/SD) & $1227.2(359)$ & $1252.89(661)$ & $1054.52(274)$ & $1213.90(664)$ & 0.37 \\
\hline Gestational age in weeks (mean/SD) & $31.4(2.9)$ & $30.5(3.9)$ & $30.0(2.8)$ & $30.0(4.1)$ & 0.10 \\
\hline Male $(n, \%)$ & $16(51.6)$ & $12(63.1)$ & $9(42.8)$ & $16(55.1)$ & 0.63 \\
\hline Maternal hypertension $(n, \%)$ & $11(35.4)$ & $4(21.0)$ & $3(14.2)$ & $3(10.3)$ & 0.10 \\
\hline Antenatal steroids ( $\mathrm{n}, \%)$ & $15(48.3)$ & $8(42.1)$ & $11(52.3)$ & $10(34.4)$ & 0.67 \\
\hline Small for gestational age $(n, \%)$ & $18(58.0)$ & $5(26.3)$ & $10(47.6)$ & $9(31.0)$ & 0.68 \\
\hline Chorioamnionitis (n, \%) & $6(19.3)$ & $11(57.8)$ & $6(28.5)$ & $12(41.3)$ & 0.04 \\
\hline Length of stay (mean/SD) & $78.5(52.8)$ & $120.2(104.9)$ & $40.9(31.1)$ & $70.7(82.5)$ & 0.63 \\
\hline
\end{tabular}

CHD: congenital heart disease; NECS: necrotizing enterocolitis (NEC) patients undergoing surgery; NECD: NEC patients undergoing peritoneal drainage; NECCAS: NEC + CHD patients undergoing surgery; NECCAD: NEC + CHD patients who were drained. ANOVA or chi-squared test. 
association between death and drainage in both groups; however, the adjusted relative risk was higher in the group without heart disease (Table 2).

We assessed the time to death in the newborns according to the presence of heart disease, type of surgical treatment, and gestational age (Figure 1) and observed that the shortest time to death occurred in the NECD group.

Table 3 shows the association between time to death and the groups. The patients submitted to drainage had

Table 2. Number (\%) of deaths in the groups and relative risk (RR) according to the presence of heart disease and type of surgical treatment.

\begin{tabular}{lrrc}
\hline Characteristics & Death & Survival & RR \\
\hline NECS N=31 (\%) & $6(19.3)$ & $25(80.6)$ & $1.45(0.66 ; 3.21)$ \\
NECCAS N=19 (\%) & $6(31.5)$ & $13(68.4)$ & $2.70(1.47 ; 4.97)$ \\
NECD N=21 (\%) & $15(71.4)$ & $6(28.5)$ & $1.97(1.08 ; 3.61)$ \\
NECCAD N=29 (\%) & $21(72.4)$ & $8(27.5)$ & $1.06(0.73 ; 1.54)$ \\
\hline
\end{tabular}

Covariates: birth weight and gestational age. CHD: congenital heart disease; NECS: necrotizing enterocolitis (NEC) patients undergoing surgery; NECD: NEC patients undergoing peritoneal drainage; NECCAS: NEC + CHD patients undergoing surgery; NECCAD: NEC + CHD patients who were drained. Simple and multiple log-binomial regression analysis.

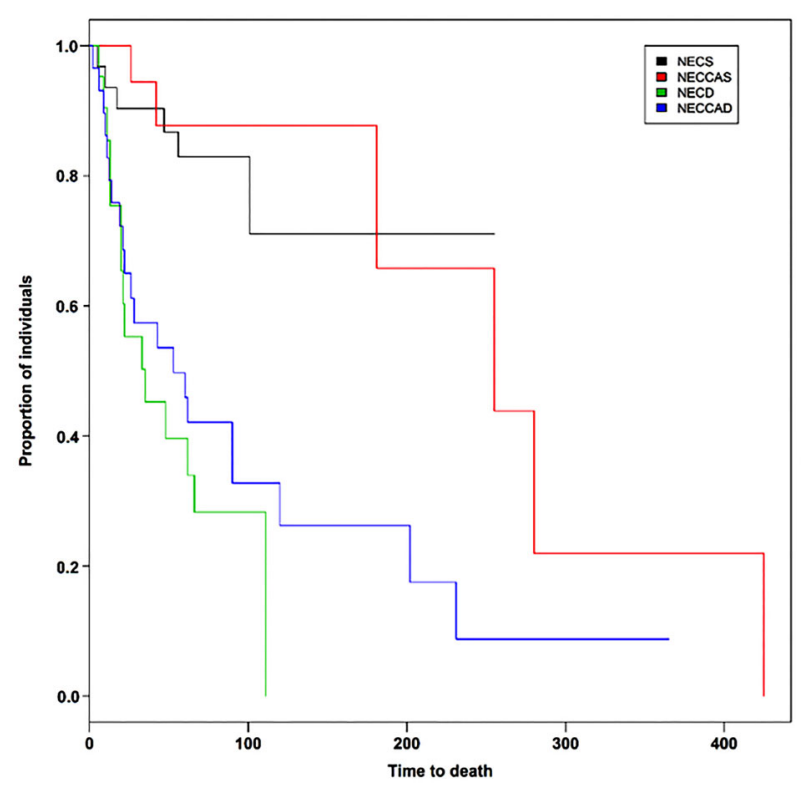

Figure 1. Time to death (days) in the groups according to the presence of heart disease and type of surgical treatment. CHD: congenital heart disease; NECS: necrotizing enterocolitis (NEC) patients undergoing surgery; NECD: NEC patients undergoing peritoneal drainage; NECCAS: NEC + CHD patients undergoing surgery; NECCAD: NEC + CHD patients who were drained.
Table 3. Association between time to death and the groups in the non-survivor patients.

\begin{tabular}{lcc}
\hline Groups & Hazard ratio $(95 \% \mathrm{Cl})$ & Hazard ratio $(95 \% \mathrm{Cl})$ - adjusted \\
\hline NECS & Reference & Reference \\
NECCAS & $0.94(0.28 ; 3.13)$ & $1.14(0.32 ; 4.09)$ \\
NECD & $6.10(2.34 ; 15.84)$ & $8.72(3.10 ; 24.54)$ \\
NECCAD & $4.21(1.69 ; 10.53)$ & $5.32(1.95 ; 14.44)$ \\
\hline
\end{tabular}

Covariates: birth weight and gestational age. CHD: congenital heart disease; NECS: necrotizing enterocolitis (NEC) patients undergoing surgery; NECD: NEC patients undergoing peritoneal drainage; NECCAS: NEC + CHD patients undergoing surgery; NECCAD: NEC + CHD patients who were drained.

the highest risk of death and their deterioration was faster compared with NECS.

\section{Discussion}

Our data demonstrated the worst outcomes in newborns submitted to drainage in both groups. Drainage did not avoid a higher incidence of death in patients with surgical NEC and clinical instability in the newborns with $\mathrm{NEC}$ or NEC + HCD.

The group characteristics were similar, and the HCD presence and the type of treatment performed did not affect the length of stay. There is a consensus that occurrence of NEC has worse outcomes in neonates with $\mathrm{CHD}$. Regarding heart diseases, the persistence of the ductus arteriosus, use of indomethacin to close the ductus, umbilical catheterization, and CHD are possible triggers for NEC development, which affects between 7 and $20 \%$ of this population $(3,14-19)$.

In a case-control study, Bubberman et al. (20) compared 18 NEC + CHD and 36 NEC patients and found different postnatal ages at the onset of symptoms, with an earlier onset in NEC+CHD. The $\mathrm{pH}$ levels were lower and the C-reactive protein levels were higher in NEC. In addition, the anatomical location was different: the colon was more frequently affected in NEC + CHD (86 vs $33 \%$ in $\mathrm{NEC}, \mathrm{P}=0.03)$. However, these authors did not find any difference in mortality between the groups (22 vs $11 \%$, respectively, $\mathrm{P}=0.47$ ).

NEC pathophysiology in cardiac patients results from low blood flow in the mesenteric vessels, a consequence of reverse flow in the abdominal aorta secondary to low diastolic pressure through the hypoxia compensatory mechanism called the "diving reflex" (15). Cardiac patients with ascites often require paracentesis, with hemodynamic improvement (16). Some types of heart disease, such as left ventricular hypoplasia, a single ventricle, coarctation of the aorta, and arteriovenous truncus, represent an even higher risk for NEC (3). The best approach to the treatment of NEC + CHD needs to be 
clarified, especially in neonates with clinical instability. Is PPD associated with better outcomes in these patients?

The conventional treatment of neonates with perforation or intestinal necrosis is laparotomy, with the creation of an ostomy. In newborns, this treatment poses a tremendous surgical risk due to the patient's critical condition (21). PPD without posterior laparotomy is related to a higher mortality and longer hospitalization than laparotomy alone (8), so its use as a definitive treatment method is not justified. Unfavorable PPD results include the presence of an intestine with necrosis and a predisposition to sepsis, which stimulates cytokine release and negatively affects the organism, deteriorating the clinical condition of the newborn (22).

Ehrlich et al. (23) analyzed the surgical procedures performed on 70 newborns weighing less than $1,000 \mathrm{~g}$ who developed NEC, and they observed a survival rate of 63 and $75 \%$ in babies that underwent PPD and laparotomy, respectively. However, patients submitted to PPD had a significantly higher number of complications. Through regression analysis, the authors concluded that the surgical choice did not influence the results. Recently, Moss et al. (24) performed a meta-analysis of studies that used both procedures to treat children with NEC. The analysis included 475 patients and showed no significant difference between laparotomy and PPD for mortality. Other studies also did not verify any significant difference in morbidity, mortality, or the length of hospital stay in newborns undergoing PPD followed by laparotomy compared with those undergoing isolated laparotomy (25). In addition, most newborns subjected to PPD required laparotomy as a second step $(11,26)$.

However, Tashiro et al. (27) reported that PPD proved useful as an initial surgical treatment in hemodynamically unstable premature patients weighing less than $1000 \mathrm{~g}$. They demonstrated patient survival was higher in NEC + CHD that underwent PPD (47\%) than in NEC patients in

\section{References}

1. Dominguez KM, Moss RL. Necrotizing enterocolitis. Clin Perinatol 2012; 39: 387-401, doi: 10.1016/j.clp.2012.04.011.

2. Wu SF, Caplan M, Lin HC. Necrotizing enterocolitis: old problem with new hope. Pediatr Neonatol 2012; 53: 158163, doi: 10.1016/j.pedneo.2012.04.001.

3. McElhinney DB, Hedrick HL, Bush DM, Pereira GR, Stafford PW, Gaynor JW, et al. Necrotizing enterocolitis in neonates with congenital heart disease: risk factors and outcomes. Pediatrics 2000; 106: 1080-1087, doi: 10.1542/peds.106.5. 1080.

4. Lau PE, Cruz SM, Ocampo EC, Nuthakki S, Candace C Style CC, Lee TC, et al. Necrotizing enterocolitis in patients with congenital heart disease: a single center experience. J Pediatr Surg 2018; 53: 914-917, doi: 10.1016/j.jpedsurg. 2018.02.014.

5. Luce WA, Schwartz RM, Beauseau W, Giannone PJ, Boettner BL, Cheatham JP, et al. Necrotizing enterocolitis general (10\%). Among patients undergoing PPD, patients with heart disease had higher survival rates. The lack of improvement in the survival of newborns diagnosed with NEC was influenced by nonspecific and often random surgical and therapeutic strategies (16).

In our study, PPD was associated with death in NECS and NECD as well as in NECCAS and NECCAD. The relative risk of death in NECCAD was higher than in NECCAS, and it was also higher in NECD compared with NECS; however, the neonates without heart disease presented a higher risk of death than newborns with heart disease. The lower risk of these patients can be explained by their more significant hypoxemia, which could partially represent ischemic preconditioning and, therefore, serve as a protective state against intestinal damage (28).

Our data found an association with faster progression to death in neonates submitted to drainage, but these data have limitations since our indication for drainage was clinical instability, and the worse outcomes are probably related to their clinical conditions. Therefore, our data are in accordance with the literature and demonstrated that drainage was ineffective in both groups and was associated with worse outcomes.

\section{Conclusion}

In conclusion, primary peritoneal drainage in neonates with or without CHD did not improve days of life, did not decrease the risk of death, and was associated with higher mortality in newborns with NEC and clinical instability.

\section{Acknowledgments}

We are grateful to Ms. Cynthia Manso for her English language assistance. L. Sbragia received a productivity scholarship from Conselho Nacional de Desenvolvimento Científico e Tecnológico (CNPq, \#302433/2014-7; Brazil). in neonates undergoing the hybrid approach to complex congenital heart disease. Pediatr Crit Care Med 2011; 12 : 46-51, doi: 10.1097/PCC.0b013e3181e3250c.

6. Hsueh W, Caplan MS, Qu XW, Tan XD, De Plaen IG, Gonzalez-Crussi F. Neonatal necrotizing enterocolitis: clinical considerations and pathogenetic concepts. Pediatr Dev Pathol 2003; 6: 6-23, doi: 10.1007/s10024-002-0602-z.

7. Raval MV, Hall NJ, Pierro A, Moss RL. Evidence-based prevention and surgical treatment of necrotizing enterocolitis-a review of randomized controlled trials. Semin Pediatr Surg 2013; 22: 117-121, doi: 10.1053/j.sempedsurg.2013. 01.009.

8. Downard CD, Renaud E, St Peter SD, Abdullah F, Islam S, Saito JM, et al. Treatment of necrotizing enterocolitis: an American Pediatric Surgical Association Outcomes and Clinical Trials Committee systematic review. J Pediatr Surg 2012; 47: 2111-2122, doi: 10.1016/j.jpedsurg.2012.08.011. 
9. Cheng W, Leung MP, Tam PK. Surgical intervention in necrotizing enterocolitis in neonates with symptomatic congenital heart disease. Pediatr Surg Int 1999; 15: 492495, doi: $10.1007 / \mathrm{s} 003830050647$.

10. Zenciroğlu A, Cakmak O, Demirel N, Baş AY, Yilmaz D, Karaman I, et al. Outcome of primary peritoneal drainage for perforated necrotizing enterocolitis: comparison between laparotomy and drainage. Eur J Pediatr Surg 2005; 15: 243247, doi: 10.1055/s-2005-865767.

11. Rees CM, Eaton S, Kiely EM, Wade AM, McHugh K, Pierro A. Peritoneal drainage or laparotomy for neonatal bowel perforation? A randomized controlled trial. Ann Surg 2008; 248: 44-51, doi: 10.1097/SLA.0b013e318176bf81.

12. Rees CM, Eaton S, Khoo AK, Kiely EM; Members of NET Trial Group, Pierro A. Peritoneal drainage does not stabilize extremely low birth weight infants with perforated bowel: data from the NET Trial. J Pediatr Surg 2010; 45: 324-328, doi: 10.1016/j.jpedsurg.2009.10.066.

13. Bell MJ, Ternberg JL, Feigin RD, Keating JP, Marshall R, Barton L, et al. Neonatal necrotizing enterocolitis. Therapeutic decisions based upon clinical staging. Ann Surg 1978; 187: 1-7, doi: 10.1097/00000658-197801000-00001.

14. Lu Q, Cheng S, Zhou M, Yu J. Risk factors for necrotizing enterocolitis in neonates: a retrospective case-control study. Pediatr Neonatol 2017; 58: 165-170, doi: 10.1016/j. pedneo.2016.04.002.

15. Siano E, Lauriti G, Ceccanti S, Zani A. Cardiogenic necrotizing enterocolitis: a clinically distinct entity from classical necrotizing enterocolitis. Eur J Pediatr Surg 2019; 29: 14-22, doi: 10.1055/s-0038-1668144.

16. Hackam DJ, Sodhi CP, Good M. New insights into necrotizing enterocolitis: From laboratory observation to personalized prevention and treatment. J Pediatr Surg 2019; 54: 398-404, doi: 10.1016/j.jpedsurg.2018.06.012.

17. Palder SB, Schwartz MZ, Tyson KR, Marr CC. Association of closure of patent ductus arteriosus and development of necrotizing enterocolitis. J Pediatr Surg 1988; 23: 422-423, doi: 10.1016/S0022-3468(88)80439-1.

18. Grosfeld JL, Chaet M, Molinari F, Engle W, Engum SA, West $\mathrm{KW}$, et al. Increased risk of necrotizing enterocolitis in premature infants with patent ductus arteriosus treated with indomethacin. Ann Surg 1996; 224: 350-357, doi: 10.1097/ 00000658-199609000-00011.
19. Kessler U, Schulte F, Cholewa D, Nelle M, Schaefer SC, Klimek PM, et al. Outcome in neonates with necrotizing enterocolitis and patent ductus arteriosus. World $\mathrm{J}$ Pediatr 2016; 12: 55-59, doi: 10.1007/s12519-015-0059-6.

20. Bubberman JM, van Zoonen A, Bruggink JLM, van der Heide M, Berge RMF, Bos AF, et al. Necrotizing enterocolitis associated with congenital heart disease: a different entity? J Pediatr Surg 2019; 54: 1755-1760, doi: 10.1016/j. jpedsurg.2018.11.012.

21. Ein SH, Marshall DG, Girvan D. Peritoneal drainage under local anesthesia for perforations from necrotizing enterocolitis. J Pediatr Surg 1977; 12: 963-967, doi: 10.1016/00223468(77)90607-8.

22. Hull MA, Fisher JG, Gutierrez IM, Jones BA, Kang KH, Kenny $M$, et al. Mortality and management of surgical necrotizing enterocolitis in very low birth weight neonates: a prospective cohort study. J Am Coll Surg 2014; 218: 11481155, doi: 10.1016/j.jamcollsurg.2013.11.015.

23. Ehrlich PF, Sato TT, Short BL, Hartman GE. Outcome of perforated necrotizing enterocolitis in the very low-birth weight neonate may be independent of the type of surgical treatment. Am Surg 2001; 67: 752-756.

24. Moss RL, Dimmitt RA, Barnhart DC, Sylvester KG, Brown $\mathrm{RL}$, Powell DM, et al. Laparotomy versus peritoneal drainage for necrotizing enterocolitis and perforation. $N$ Engl $J$ Med 2006; 354: 2225-2234, doi: 10.1056/NEJMoa054605.

25. Neu J, Walker WA. Necrotizing enterocolitis. N Engl J Med 2011; 364: 255-264, doi: 10.1056/NEJMra1005408.

26. Stey A, Barnert ES, Tseng CH, Keeler E, Needleman J, Leng $M$, et al. Outcomes and costs of surgical treatments of necrotizing enterocolitis. Pediatrics 2015; 135: e1190e1197, doi: 10.1542/peds.2014-1058.

27. Tashiro J, Wagenaar AE, Perez EA, Sola JE. Peritoneal drainage is associated with higher survival rates for necrotizing enterocolitis in premature, extremely low birth weight infants. J Surg Res 2017; 218: 132-138, doi: 10.1016/j. jss.2017.05.064.

28. Hummitzsch L, Zitta K, Berndt R, Wong YL, Rusch R, Hess $\mathrm{K}$, et al. Remote ischemic preconditioning attenuates intestinal mucosal damage: insight from a rat model of ischemia-reperfusion injury. $J$ Transl Med 2019; 17: 136, doi: 10.1186/s12967-019-1885-4. 